\title{
Systematic review of the effects of oral nutritional interventions in care homes
}

\author{
E. L. Parsons, R. J. Stratton and M. Elia \\ Institute of Human Nutrition, University of Southampton, Southampton SO16 6YD, UK
}

There is some uncertainty about the effectiveness of different forms of oral nutrition support in the management of malnutrition in care homes. A systematic review was undertaken to investigate the evidence for the use of oral nutritional interventions (dietary advice, food fortification, oral nutritional supplements (ONS)) on nutritional, functional and clinical outcomes in care home residents.

The systematic review was undertaken following accepted methodology, including the systematic searching of databases (PubMed, $\mathrm{CAB}$ abstracts, Ovid and Embase) and bibliographies (up to December 2009). A total of 16 randomised controlled trials (RCT) in care homes ( $n$ 826) were identified that compared either food fortification ( 8 RCT, $n$ 364) or ONS ( 7 RCT, $n$ 406) with routine care. No trials of dietary advice $v$. routine care were found and $4 \mathrm{RCT}(n$ 127) compared food fortification with ONS. The duration of intervention was $4 \mathrm{~d}$ to 1 year. Food fortification strategies included the use of energy-rich ingredients and food snacks. ONS were mostly multi-nutrient, ready-made liquids. Outcomes assessed were; energy intake, body weight, body function (e.g. quality of life and muscle strength) and clinical outcomes (e.g. infections). Analysis was undertaken using Meta-analysis (Comprehensive Meta-Analysis v2.0) where possible.

One food fortification trial reported small, non-significant changes in energy intake. Few food fortification trials reported functional outcomes, with no significant differences being observed and there was insufficient data for meta-analysis. No food fortification trials reported clinical outcomes. Combined analysis of 3 ONS RCT ( $n$ 196) showed improvements in energy intake (mean difference $514.63 \mathrm{~kJ}$ $(123 \mathrm{kcal})(95 \%$ CI 92,154$) \mathrm{kcal}, P<0.0001$. Meta-analysis of 3 ONS RCT $(n$ 195) found a significant difference in body weight $(1.7$ $(95 \%$ CI $0.8,2.6) \mathrm{kg}, P<0.001$, random effects model). No studies reported significant functional changes. One RCT reported significant reductions in infections (ONS; $53 \%$ - no infections, control; $44 \%$ - no infections, $P=0.001$ (calculated using published data)), and bed days (ONS: $7.5 \pm 2.1 v$. control: $17.3 \pm 5.6, P<0.001$ (calculated using published data)) over 12 months of ONS and another reported improvements in pressure ulcer healing with 8 weeks of ONS (change in pressure ulcer score; $5.56 v .2 .85, P<0.05$ ). There was insufficient functional and clinical outcome data for meta-analysis. The one RCT of food snacks $v$. ONS reported significantly greater nutritional intakes with ONS (mean difference $1150.6 \mathrm{~kJ}(275 \mathrm{kcal}), P<0.01)$ but no functional or clinical outcomes were measured.

This systematic review of studies in care homes suggests that: (i) there is extremely little evidence for the use of food fortification and no evidence for dietary advice; (ii) there is some evidence that ONS can improve energy intake, body weight and clinical outcomes. More research in well-designed trials is needed to investigate the effects of different kinds of oral nutritional support in care homes.

An unrestricted educational grant from Nutricia. 